October 1997

\title{
Comments on the Problem of a Covariant Formulation of Matrix Theory
}

\author{
H.AWATA and D.MINIC \\ Enrico Fermi Institute \\ The University of Chicago \\ Chicago, IL 60637 \\ and \\ Physics Department \\ Pennsylvania State University \\ University Park, PA 16802 \\ awata@yukawa.uchicago.edu,minic@hbar.phys.psu.edu
}

\begin{abstract}
A possible avenue towards the covariant formulation of the bosonic Matrix Theory is explored. The approach is guided by the known covariant description of the bosonic membrane. We point out various problems with this particular covariantization scheme, stemming from the central question of how to enlarge the original $U(N)$ symmetry of Matrix Theory while preserving all of its essential features in the infinite momentum frame.
\end{abstract}


Matrix theory, unlike its classical counterpart - membrane theory [1], is currently formulated only in a background dependent way [2]. This article addresses the question of whether a covariant formulation of the bosonic part of Matrix theory is possible which emulates the well-known covariant description of the bosonic membrane. (Although we do not discuss supersymmetry in this paper, our analysis is motivated in part by the fact that a covariant formulation of the full supersymmetric Matrix theory would circumvent the puzzling issue of longitudinal boost invariance, and point out a way towards a covariant description of the Matrix theory five-brane.)

The basic idea of this note is to take the existing covariant action for a $d+1$ dimensional bosonic membrane [1] (with, let's say, $d=10$ ), which possesses the full three-dimensional world-volume diffeomorphism invariance, and which in the light-cone gauge leads to a Hamiltonian that is invariant under the residual area preserving diffeomorphisms of the transverse membrane, and examine its matrix analog. The light-cone membrane Hamiltonian coincides with the bosonic Matrix Theory Hamiltonian [2], upon application of the Goldstone-Hoppe prescription [3]. This prescription can be interpreted as a regularization of the residual area preserving diffeomorphisms in terms of the $U(N)(N=\infty)$ rotations of matrices [3], the transverse spatial coordinates being mapped into $N \times N$ hermitian matrices, which describe the transverse coordinates of $N D$ 0-branes (the $U(N)$ symmetry governs the statistics of a system of $N D 0$-branes [2],[4]).

The following natural question arises of whether it is possible to implement an analogous regularization procedure in the original covariant description of the bosonic membrane. Then, in case one is fortunate enough to start with a regularized covariant theory with sufficiently large symmetry (some appropriate matrix analog of the three-dimensional diffeomorphisms) one could study the light-cone gauge and determine whether the light- 
cone Hamiltonian coincides with the bosonic Matrix theory Hamiltonian.

The note is organized in three parts: To start with, we review some well known facts about the covariant formulation of the bosonic membrane following the classic reference [1]. We then present a possible route towards covariant formulation of Matrix theory guided by the analogy with the covariant membrane dynamics. Finally, we discuss various problems with this approach, all of which are rooted in the crucial question of how to extend the original $U(N)$ symmetry of Matrix theory (or how to regularize the full three-dimensional diffeomorphisms of the covariant membrane formulation) and be able to recover the infinite momentum frame description upon gauge fixing. We also compare our results with Smolin's recent analysis of the question of the covariant quantization of membrane dynamics [6].

Let us then start from the familiar bosonic membrane action [1]

$$
S=-\int d^{3} \xi \sqrt{-\operatorname{detg}_{i j}}
$$

where the induced world-volume metric $g_{i j}$ is

$$
g_{i j}=\partial_{i} x_{\mu} \partial_{j} x_{\nu} \eta^{\mu \nu}
$$

and where $x_{\mu}(\mu=0,1, \ldots, d)$ denote the target space coordinates of a $d+1$ dimensional bosonic membrane. $x_{\mu}$ 's are functions of the three world-volume coordinates $\xi^{i}, i=0,1,2$.

The world-volume reparametrization invariance $\delta x_{\mu}=\epsilon^{i} \partial_{i} x_{\mu}$ allows one to go to lightcone gauge and rewrite the theory in terms of physically relevant transverse variables. The light-cone coordinates are defined as

$$
x_{ \pm}=\frac{1}{\sqrt{2}}\left(x_{d} \pm x_{0}\right)
$$

and the light-cone gauge

$$
\partial_{i} x_{+}=\delta_{i 0}
$$


The world volume coordinates $\xi^{i}$ split as

$$
\left(\xi^{0}, \xi^{s}\right) \rightarrow\left(t, \xi^{s}\right)
$$

The Lagrangian density reads as follows (we adopt the notation found in [1])

$$
\mathcal{L}=-\sqrt{g \Delta}
$$

where $g \equiv \operatorname{det}_{r s}(r, s=1,2)$ is the determinant of the induced two dimensional metric, and $g$ and $\Delta$ are determined by

$$
\begin{aligned}
g & =\frac{1}{2}\left\{x_{a}, x_{b}\right\}^{2} \\
\Delta & =-g_{00}+u^{r} u_{r} \equiv-\left|D_{0} x_{\mu}\right|^{2} .
\end{aligned}
$$

Here $D_{0} \equiv \partial_{0}-u^{r} \partial_{r}$ and $u^{r}=g^{r s} u_{s}\left(u_{r} \equiv g_{0 r}\right.$ plays the role analogous to that of the shift function in the Hamiltonian approach to general relativity), $a=1, \ldots, d-1$ stands for the index of the transverse space, and $\{$,$\} denotes the usual Poisson brackets with respect to$ $\xi_{s}$

$$
\left\{x_{a}, x_{b}\right\} \equiv \partial_{\xi^{1}} x_{a} \partial_{\xi^{2}} x_{b}-\partial_{\xi^{2}} x_{a} \partial_{\xi^{1}} x_{b}
$$

Furthermore, in the light-cone gauge $(3 \mathrm{~b})$

$$
\begin{aligned}
g_{00} & =2 \partial_{0} x_{-}+\left(\partial_{0} x_{a}\right)^{2} \\
u_{r} & =\partial_{r} x_{-}+\partial_{0} x_{a} \partial_{r} x_{a} .
\end{aligned}
$$

The conjugate momenta are easily calculated

$$
\begin{aligned}
& p_{a}=\sqrt{\Delta^{-1} g}\left(\partial_{0} x_{a}-u^{r} \partial_{r} x_{a}\right)=\sqrt{\Delta^{-1} g} D_{0} x_{a} \\
& p_{+}=\sqrt{\Delta^{-1} g}
\end{aligned}
$$

Then the light-cone Hamiltonian density $\mathcal{H}=p_{a} \partial_{0} x_{a}+p_{+} \partial_{0} x_{-}-\mathcal{L}$ reads simply as follows

$$
\mathcal{H}=\frac{1}{2 p_{+}}\left(p_{a}{ }^{2}+\frac{1}{2}\left\{x_{a}, x_{b}\right\}^{2}\right) .
$$


From this form of the light cone Hamiltonian it is seen that the original three-volume diffeomorphisms $\delta x_{\mu}=\epsilon^{i} \partial_{i} x_{\mu}$ reduce to the area preserving diffeomorphisms described by $\delta x_{a}=\epsilon^{r s} \partial_{r} w \partial_{s} x_{a}=\left\{w, x_{a}\right\}$. The longitudinal coordinate $x_{-}$does not appear explicitly in (8) and is determined from the primary constraint $p_{a} \partial_{r} x_{a}+p_{+} \partial_{r} x_{-} \sim 0$ and the requirement that the longitudinal momentum is time-independent $\partial_{0} p_{+}=0$, or essentially, the gauge condition $u^{r}=0[1]$.

At this point the light-cone Hamiltonian is regularized by applying the GoldstoneHoppe map between representation theories of the algebra of the area preserving diffeomorphisms and the $N=\infty$ limit of Lie algebras [3]. The Goldstone-Hoppe prescription instructs us to perform the following translation of the transverse spatial coordinates

$$
\left\{x_{a}, x_{b}\right\} \rightarrow\left[X_{a}, X_{b}\right]
$$

where now $X_{a}$ denote large $N \times N$ hermitian matrices (the area preserving diffeomorphisms are mapped into $U(N)$ rotations of matrices for the simplest case of a spherical membrane). Also

$$
\int d \xi^{1} d \xi^{2} \rightarrow T r
$$

The resulting regularized Hamiltonian density is precisely the bosonic part of the Matrix theory Hamiltonian [2]. (The bosonic membrane thus represents a classical configuration of the bosonic part of Matrix theory.)

After this short review let us return to the original covariant bosonic membrane action (1) and try to apply the Goldstone-Hoppe regularization at the very covariant level. (The covariant formulation of the bosonic part of Matrix theory should thus contain the covariant bosonic membrane as a natural classical configuration.) First, we notice that the three by three determinant in (1) can be expanded in such a way so that there appears a term of 
the form

$$
\frac{1}{2}\left(\partial_{t} x_{\mu}\right)^{2}\left\{x_{\rho}, x_{\nu}\right\}^{2}
$$

and two other terms which can be collected together to read $\left(\xi^{1} \equiv \sigma, \xi^{2} \equiv \tau\right)$

$$
-\left(\partial_{\sigma} x_{\nu}\left(\partial_{t} x^{\mu} \partial_{\tau} x_{\mu}\right)-\partial_{\tau} x_{\nu}\left(\partial_{t} x^{\mu} \partial_{\sigma} x_{\mu}\right)\right)^{2}
$$

In other words, the original Nambu-Goto type action for the bosonic membrane (1) can be written in the so-called Barbour-Bertotti form [5], [6]

$$
S=-\int d^{3} \xi \sqrt{-\frac{1}{2} \dot{x}^{\mu} \dot{x}^{\nu}\left(\eta_{\mu \nu} g-2\left\{x_{\mu}, x_{\beta}\right\}\left\{x_{\nu}, x^{\beta}\right\}\right)} .
$$

Here $\dot{x}_{\mu} \equiv \partial_{t} x_{\mu}$. This action naturally incorporates time reparametrization invariance on the world-volume of the membrane.

The application of the original Goldstone-Hoppe dictionary to (10c) then leads (at least naively) to the following regularized action

$$
S_{M}=-T r \int d t \sqrt{-\frac{1}{2}\left(\dot{X}_{\mu}\right)^{2}\left[X_{\mu}, X_{\nu}\right]^{2}+\left(\dot{X}_{\mu}\left[X_{\mu}, X_{\nu}\right]\right)^{2}},
$$

where the Poisson brackets with respect to $\xi^{s}$ get replaced by the commutators of timedependent matrices and the integral over $\xi^{s}$ by the usual matrix trace $\operatorname{Tr} . X_{\mu}(t)(\mu=$ $0, \ldots, d)$ represent now large $N \times N$ hermitian matrices; $t$ plays the role of a "world-line" parameter. Obviously, to properly define this expression one has to examine the question of ordering. One way to do this is to symmetrize all matrix products. Another question pertains to the definition of $\sqrt{M}$, where $M$ is an $N \times N$ matrix. We use one of the formal expressions, for example, $\sqrt{M} \equiv \exp \left(\frac{1}{2} \log M\right)$ or $\sqrt{M} \equiv(1-(1-M))^{1 / 2}=1-\frac{1}{2}(1-M)+\ldots$, so that $M^{n} M^{m}=M^{n+m}$ and $\left(u M u^{-1}\right)^{n}=u M^{n} u^{-1}$, for some unitary $N \times N$ matrix $u$.

What are the symmetries of (11)? One immediately sees that apart from "world-line" $t$-reparametrization invariance, $(11)$ is endowed with the following symmetry

$$
X_{\mu} \rightarrow u X_{\mu} u^{-1}+f_{\mu}
$$


where $u$ is a unitary $t$-independent matrix (describing unitary time-independent rotations), and $f_{\mu} \propto \mathbf{1}$ (1 is the unit $N \times N$ matrix) are constant matrices (describing constant shifts). (Notice the similarity of (12) to a global, time-independent, Poincare invariance.) It can be seen that (12) together with $t$-reparametrization symmetry is not enough to try to go to light-cone gauge i.e. set $X_{+} \propto \mathbf{1} ;$ here $X_{ \pm} \equiv \frac{1}{\sqrt{2}}\left(X_{d} \pm X_{0}\right)$.

However, there exists another natural expression for $S_{M}$ which leads to completely equivalent physical results in the continuum membrane limit, namely

$$
S_{M}=-\int d t \operatorname{Tr} \sqrt{-\frac{1}{2}\left(D_{0} X_{\mu}\right)^{2}\left[X_{\nu}, X_{\rho}\right]^{2}}
$$

where $D_{0} \equiv \partial_{0}-[\omega,$.$] . Eq. (13) follows from eqs. (1) - (6) and the Goldstone-Hoppe$ dictionary. $\omega$ is the matrix analog of the shift function $u^{r}$ in (5). (Observe that both (11) and (13) are natural on dimensional grounds.) However, (13) is invariant under

$$
X_{\mu}(t) \rightarrow u(t) X_{\mu}(t) u^{-1}(t)+f_{\mu}
$$

in addition to "world-line" $t$-reparametrization invariance $t \rightarrow \phi(t)$ (note that $u$ is now a time-dependent matrix; $f_{\mu} \propto \mathbf{1}$ as before). The first term in (14) describes time-dependent $U(N)$ rotations and the second - translations. Also

$$
\omega \rightarrow u(t) \omega u^{-1}(t)+\left(\partial_{0} u\right) u^{-1}
$$

Given (14) we can immediately diagonalize $X_{+}$

$$
X_{+} \sim \operatorname{diag}\left(\phi_{1}(t), \ldots, \phi_{N}(t)\right)
$$

Now comes the crucial point. One could expect that in the large N limit all of $\phi_{i}(t) \rightarrow \phi(t)$. Not being able to prove this statement, we take it as a crucial assumption and proceed with the computation of the light-cone Lagrangian and Hamiltonian! If we assume that 
$\phi_{i}(t) \rightarrow \phi(t)$, by using $t$-reparametrization invariance we can set $\phi(t) \rightarrow t$ (therefore recovering the global time of Matrix Theory in the infinite momentum frame), so that finally the gauge condition reads

$$
X_{+}=\left(X_{+}(0)+t\right) \mathbf{1}
$$

This equation would define the matrix version of the light-cone gauge (3b).

We can further fix the gauge (by utilizing (14a) and letting $\omega=0$, the matrix analog of $u^{r}=0$ gauge [1]) thus ending up with

$$
S_{M}=-\int d t \operatorname{Tr} \sqrt{-\frac{1}{2}\left(\partial_{0} X_{\mu}\right)^{2}\left[X_{\nu}, X_{\rho}\right]^{2}} .
$$

$\left(\right.$ Here $\left(\partial_{0} X_{\mu}\right)^{2}=2 \dot{X}_{-}+\left(\dot{X}_{a}\right)^{2}$ and $\left[X_{\mu}, X_{\nu}\right]^{2}=\left[X_{a}, X_{b}\right]^{2}$.) This action is seen to be invariant under the residual transformations

$$
X_{a}(t) \rightarrow \lambda X_{a}(t) \lambda^{-1}+f_{a}
$$

where $\lambda$ is again a constant unitary matrix and $f_{a} \propto \mathbf{1}$.

To summarize: Starting from a covariant description of M-theory of a Nambu-Goto type (13) in terms of $d+1$ time-dependent matrices, which is invariant under timedependent $U(N)$ rotations of matrices and constant translations (14) as well as "worldline" $t$-reparametrizations, and by naively diagonalizing one of the eleven matrices, in order to define the matrix analog of the "light-cone" gauge (15a,b) (which appears to be possible only in the large $N$ limit ), we end up with (16). (In essence, we propose to regularize the volume preserving part of the full three-dimensional diffeomorphisms describing the covariant membrane dynamics by $t$-dependent $U(N)$ rotations and "worldline" $t$-reparametrizations in the $N=\infty$ limit. We argue that the light-cone gauge fixing is possible only in the large $N$ limit. Upon gauge fixing we find that the light-cone Lagrangian is invariant under the residual symmetry (17).) 
At this point we can (at least formally) rewrite the Lagrangian density $\mathcal{L}_{M}$ given by (16) as

$$
\mathcal{L}_{M} \equiv-\sqrt{\Delta} \sqrt{g}
$$

Here we adopt a particular ordering prescription for the Lagrangian, conjugate momenta and Hamiltonian, in order to make contact with the Matrix theory Hamiltonian description. The matrices $g$ and $\Delta$ are defined as follows

$$
\begin{aligned}
g & =\frac{1}{2}\left[X_{\mu}, X_{\nu}\right]^{2}=\frac{1}{2}\left[X_{a}, X_{b}\right]^{2} \\
\Delta & =-\left(D_{0} X_{\mu}\right)^{2} \rightarrow-\left(\partial_{0} X_{\mu}\right)^{2}=-2 \dot{X}_{-}-\left(\dot{X}_{a}\right)^{2} .
\end{aligned}
$$

and the conjugate momenta

$$
\begin{aligned}
P_{a} & \equiv \dot{X}_{a} \frac{1}{\sqrt{\Delta}} \sqrt{g} \\
P_{+} & \equiv \frac{1}{\sqrt{\Delta}} \sqrt{g} .
\end{aligned}
$$

Using these formulae for the conjugate momenta we can evaluate the light-cone Hamiltonian $\mathcal{H}_{M} \equiv \dot{X}_{a} P_{a}+\dot{X}_{-} P_{+}-\mathcal{L}_{M}$ which equals $-\dot{X}_{-} \frac{1}{\sqrt{\Delta}} \sqrt{g}$ or

$$
\mathcal{H}_{M}=\frac{1}{2 P_{+}^{\dagger}}\left(\left|P_{a}\right|^{2}+\frac{1}{2}\left[X_{a}, X_{b}\right]^{2}\right)
$$

We have defined $\left|P_{a}\right|^{2} \equiv P_{a}^{\dagger} P_{a}, \frac{1}{P_{+}^{\dagger}} \equiv \sqrt{\Delta} \frac{1}{\sqrt{g}}$ and used the fact that

$$
\left(\left(\dot{X}_{a}\right)^{2}+\dot{X}_{-}+\frac{1}{2} \Delta\right) \frac{1}{\sqrt{\Delta}} \sqrt{g}=\frac{1}{2} \sqrt{\Delta} \frac{1}{\sqrt{g}}\left|P_{a}\right|^{2} .
$$

Note that the longitudinal matrix coordinate $X_{-}$is eliminated via $2 \dot{X}_{-}=-\left(\Delta+\left(\dot{X}_{a}\right)^{2}\right)$. (Notice also that eqs. (18) - (21) resemble the analogous expressions in the continuum case, namely eqs. (4), (5), (7) and (8).) The expression (21) agrees with the bosonic part of the Matrix theory Hamiltonian provided that it is possible to take $P_{+}$outside the trace (essentially by stating that $P_{+} \propto \mathbf{1}$ ), and by treating $P_{a}$ as the conjugate momentum of $X_{a}$. Furthermore, Matrix theory demands that the longitudinal momentum $P_{+}$be quantized 
$P_{+}=N / R, R$ being the extent of the compact longitudinal direction. How do we justify $P_{+} \propto 1$ ? One could argue that by rescaling the light-cone Hamiltonian (21) by $\frac{1}{\sqrt{\Delta}} \sqrt{g}$, the longitudinal momentum $P_{+} \propto \mathbf{1}$, so that $P_{+}$can be taken outside the trace. One can then take the rescaled Hamiltonian to be the light-cone Hamiltonian, and define $P_{a}$ to be conjugate to $X_{a}$, thus making contact with Matrix theory in the infinite momentum frame. Naturally, if the eigenvalues of the longitudinal direction are all of order $R$, then

$$
P_{+} \sim \frac{1}{R} \mathbf{1}
$$

In other words, $\operatorname{Tr} P_{+} \sim N / R$ (where $N$ defines the number of $D 0$-branes as in Matrix theory).

Let us recapitulate what we have done: By taking the existing covariant formulation of the bosonic membrane we have attempted to formulate a covariant description of the bosonic part of Matrix theory. The crucial issue was to come up with a well-motivated guess for the extension of the original $U(N)$ symmetry that characterizes the infinite momentum frame limit of Matrix theory. Guided by the fact that the infinite momentum frame membrane dynamics is determined by the residual area preserving, or symplectic, diffeomorphisms, which form a subset of the original full three-dimensional diffeomorphisms of the classical covariant membrane action, we have enlarged the original $U(N)$ symmetry of Matrix theory by including "world-line" $t$-reparametrization invariance, thereby eliminating the global time that is one of the defining features of the infinite momentum frame formulation of Matrix theory. It seems that it is necessary to let $N \rightarrow \infty$ in order to define the matrix analog of the light-cone gauge (15) and recover the global time.

The crucial question arises: Can we really fix the gauge in the large $N$ limit as indicated by (15)? In other words, do we really have enough symmetry to go to what we call matrix analog of the light-cone gauge? These questions are crucially related to the problem 
of a proper regularization of the original classical three-dimensional diffeomorphisms (also emphasized by M. Li and T. Yoneya in an unpublished work [7]). As recently pointed out by Smolin [6], the area preserving diffeomorphisms are realized linearly in the classical membrane theory, and they nicely map into $U(N)$ gauge transformations in Matrix theory (viewed as the quantization of the light-cone membrane theory). However, the non-area preserving diffeomorphisms, i.e. original three-dimensional diffeomorphisms modulo area preserving diffeomorphisms, are realized non-linearly, and it is their regularization that is essential for the problem of regularization of the full three-dimensional world-volume diffeomophisms. In the approach considered in this article the non-area preserving diffeomorphisms are not explicitly taken into account.

Therefore it seems that in order to answer the crucial questions pertaining to the problem of a covariant formulation of the bosonic Matrix theory, to wit: the enlargement of the original $U(N)$ gauge symmetry (or put differently, the proper "quantization" of the three-dimensional diffeomorphisms of the covariant formulation of the bosonic membrane), the issue of gauge fixing and recovery of the $U(N)$ invariance in the infinite momentum limit, the decoupling of the longitudinal matrix coordinate $X_{-}$, and the quantization of the longitudinal momentum $P_{+}$, one should first understand the question of "quantization" of the non-area preserving diffeomorphisms in the classical membrane theory. It is not clear at the moment how any of the above issues are affected by supersymmetry, which is surely the most important aspect of the physics of Matrix theory in its current form. (We add that we do not know whether the question of locality can be addressed in the present approach, nor whether can one construct many-body states from block diagonal matrices as in [2]. Also it is not clear what kind of objects replace partons in the covariant approach, and what role, if any, is played by the holographic principle [2], [8].) 
Finally, let us briefly note that the real covariant formulation of Matrix theory should naturally incorporate the space-time uncertainty principle of Yoneya [9], and Li and Yoneya [10], which is known to hold both in string theory and Matrix theory. Within the approach outlined above the space-time uncertainty principle should be expected to come out from the following commutator

$$
\left[X_{+}, X_{a}\right] \sim l^{2},
$$

where $l$ denotes the fundamental unit of length. The expression (23) naturally leads to

$$
\delta t \delta x \sim l^{2},
$$

which represents the usual formulation of the space-time uncertainty principle [9],[10].

\section{Acknowledgements}

We are grateful to T. Banks, P. Berglund, S. Chaudhuri, J. Harvey, M. Gunaydin, A. Klemm, R.Leigh, J. Polchinski, M. Rozali, P. Pouliot, L. Smolin and especially M. Li and T. Yoneya for many important discussions. D. M. wishes to thank the Institute for Theoretical Physics, Santa Barbara and Enrico Fermi Institute of the University of Chicago, for providing such stimulating working environments during the time when most of this work was done. We are deeply indebted to S. Chaudhuri for many useful suggestions regarding the original version of this note. This work is supported in part by Grant-in-Aid for Scientific Research from Ministry of Science and Culture.

\section{References}

1. For references consult B. de Wit, J. Hoppe and H. Nicolai, Nucl. Phys. B305 (1988) 545.

2. T. Banks, W. Fischler, S. H. Shenker and L. Susskind, Phys. Rev. D55 (1997) 5112. For a review and further references, see T.Banks, "Matrix Theory", hep-th/9710231. 
3. J. Goldstone, unpublished; J. Hoppe, MIT Ph.D. thesis, 1982 and in "Proc. Int. Workshop on Constraint's Theory and Relativistic Dynamics", G. Longhi and L. Lusanna, eds. (World Scientific, 1987); J. Hoppe, Int. J. Mod. Phys. A4 (1989) 5235; D. Fairlie, P. Fletcher and C. Zachos, J. Math. Phys. 31 (1990) 1088.

4 J. Polchinski, Phys. Rev. Lett. 75 (1995) 4724; J. Polchinski, S. Chaudhuri and C. V. Johnson, "Notes on $D$-branes, hep-th/9602052; J. Polchinski, "TASI Lectures on D-branes", hep-th/9611050; E. Witten, Nucl. Phys. B460 (1995) 338; M. Douglas, D. Kabat, P. Pouliot, S, Shenker, Nucl. Phys. B485 (1997) 85.

5. J. B. Barbour, Nature 249 (1974) 328 (Erratum, Nature 250 (1974) 606); Nuovo Cimento 26B (1975) 16; J. B. Barbour and B. Bertotti, Nuovo Cimento 38B (1977) 1; Proc. Roy. Soc. Lond. A 382 (1982) 295.

6. L. Smolin, "Covariant Quantization of Membrane Dynamics", hep-th/9710191.

7. M. Li and T. Yoneya, private communication.

8. L. Susskind, J. Math. Phys. 36 (1995) 6377; G. 't Hooft, "Dimensional Reduction in Quantum Gravity". gr-qc/9310026.

9. T. Yoneya, Mod. Phys. Lett. A4 (1989) 1587. See also T. Yoneya, in "Wandering in the Fields". K. Kawarabajashi and A. Ukawa, eds. (World Scientific, 1987) pp.419; and "Quantum String Theory", N. Kawamoto and T. Kugo, eds. (Springer, 1988) pp.23.

10. M. Li and T. Yoneya, Phys. Rev. Lett. 78 (1997) 1219. 